\title{
PAGAMENTO POR SERVIÇO AMBIENTAL EM FLORESTA OMBRÓFILA DENSA SECUNDÁRIA NO SUDESTE DO PARÁ
}

\author{
PAYMENT FOR ENVIRONMENTAL SERVICE IN SECONDARY DENSE OMBROPHYLOUS \\ FOREST IN THE SOUTHEAST OF THE PARÁ STATE
}

\author{
Willian Santos Paiva ${ }^{1}$, Gleiciane Cardoso Costa Camelo ${ }^{1}$, Raquel Feitosa de Araújo ${ }^{1}$, \\ Selma Lopes Goulart ${ }^{1}$, Simone Filipini Abrão ${ }^{2}$, Ângelo Augusto Ebling ${ }^{1}$ \\ ${ }^{1}$ Universidade Federal Rural da Amazônia, Parauapebas, Pará, Brasil - willianPaiva7@hotmail.com, \\ gleiciane.gc9@gmail.com, raquel.feitosa@live.com, lopesgoulart@yahoo.com.br \& \\ aebling@hotmail.com \\ ${ }^{2}$ Universidade Federal do Paraná, Curitiba, Paraná, Brasil-simone_abrao@hotmail.com
}

\section{RESUMO}

Os pagamentos por serviços de sequestro e estocagem de carbono surgiram como uma forma de combater o desmatamento. Desta forma, o objetivo deste estudo foi avaliar o potencial que um remanescente florestal secundário, localizado na região sudeste do estado do Pará, possui em receber pagamentos por serviços ambientais (PSA) pelo sequestro e armazenamento de carbono. Para a quantificação de carbono na serapilheira, foram instalados coletores no remanescente florestal para posterior determinação do teor de carbono nos diferentes componentes e relacionar a produção com as variáveis climáticas. O carbono, incremento de carbono e carbono estocado no remanescente foram obtidos por meio de informações coletadas em inventário florestal contínuo e utilização de fatores de conversão disponíveis na literatura. A partir do teor médio de carbono de cada componente da serapilheira e do volume de madeira estocado na floresta, observou-se que o remanescente apresenta $25,45 \mathrm{Mg}$. ha-1 de carbono estocados na sua biomassa viva e apresenta um incremento de carbono de 1,98 Mg.ha-1.ano. 0 teor de carbono médio dos componentes da serapilheira foi de $47,61 \%$, apresentando incremento anual de carbono igual a 2,73

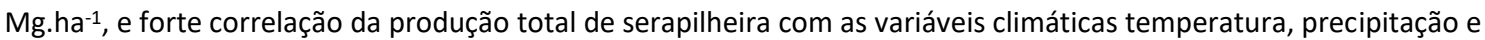
umidade relativa. Utilizando como base os valores pagos pela tonelada de carbono sequestrado, estimou-se que o remanescente florestal estudado poderia receber $859,56 €$.ha-1, sendo que este valor poderia servir de incentivo para a conservação e manutenção da biodiversidade pelo seu custo de oportunidade.

PALAVRAS-CHAVE: Conservação da biodiversidade, Floresta Amazônica, Políticas públicas, Serviços ambientais

\section{ABSTRACT}

Payments for carbon sequestration and storage services have emerged as a way to combat deforestation. Thus, the objective of this study was to evaluate the potential that a secondary forest remnant, located in the southeastern region of the state of Pará, has in receiving payments for environmental services for carbon sequestration and storage. To quantify carbon in the litter, collectors were installed in the forest remnant, for subsequent determination of the carbon content in the different components and to relate the production with the climatic variables. Carbon increment and carbon stored in the remnant were obtained through information collected from continuous forest inventory and using conversion factors available in the literature. From the average carbon content of each litter components and the stocked volume of the forest it was observed that the remnant has $25.45 \mathrm{Mg}$. ha-1 carbon stored in its living biomass and has a carbon increment of $1.98 \mathrm{Mg}^{-h^{-1}}$.year. The average carbon content of the litter


litter production and temperature, rainfall and relative humidity. Based on the amounts paid per tonne of carbon sequestered, it was estimated that the studied forest remnant could receive $€ 859.56 \mathrm{ha}$, and this value could serve as an incentive for the conservation and maintenance of biodiversity at your opportunity cost.

KEYWORDS: Amazon Rainforest, Conservation of biodiversity, Environmental services, Public policy 


\section{INTRODUÇÃO}

Desde o fim dos anos 1990 os pagamentos por serviços ambientais (PSA) ganharam destaque em inovação nas políticas ambientais praticadas, como um instrumento de mercado para viabilizar a proteção ambiental, tornando-se uma opção viável para a melhoria da gestão do meio ambiente (MATTEI \& ROSSO, 2014; WUNDER et al. 2009).

Chan et al. (2017) afirmam que os desafios advindos da sustentabilidade e da conservação da biodiversidade requerem soluções baseadas em ações de mercado. O PSA, juntamente com outros acordos desta natureza, resultou na "recuperação do ambientalismo" tido como derrotado mediante as ameaças constantes aos ecossistemas e aos serviços provindos destes.

Os PSAs podem ser projetos locais ou de caráter expansivo, geográfico e monetariamente. A exemplo deste último, cita-se os investimentos europeus em combate ao desmatamento e em incentivo a recuperação de áreas florestais na Amazônia brasileira (CHAN et al. 2017).

Em 2005 foi apresentada pela Organização das Nações Unidas (ONU) a Avaliação Ecossistêmica do Milênio (AEM), um estudo realizado com o objetivo de avaliar as consequências das mudanças nos ecossistemas sobre o bem-estar humano e estabelecer uma base científica que fundamentasse as ações necessárias para assegurar a conservação e o uso sustentável dos ecossistemas, bem como suas contribuições para o bem-estar humano (UN, 2005). A AEM classificou os serviços ambientais em serviços de provisão (os produtos obtidos dos ecossistemas), serviços de suporte (contribuem para a produção de outros serviços ecossistêmicos), serviços culturais (benefícios intangíveis obtidos, de natureza educacional, religiosa ou estético-paisagística e serviços de regulação (benefícios obtidos a partir de processos naturais que regulam as condições ambientais). A Floresta Amazônica, maior floresta tropical do mundo, assume papel importante na prestação de todos estes serviços, essencialmente sobre os serviços de regulação, que englobam, entre outros, os processos de sequestro e estocagem de carbono.

O domínio Amazônico estrutura-se de maneira primordial no ciclo global de carbono, influenciando nos padrões e mudanças climáticas, a nível de planeta, pela capacidade liberação e, principalmente, de absorção de $\mathrm{CO}_{2}$ por meio do desenvolvimento da vegetação incidente (OTUKEl et al. 2015; SOUZA et al. 2014). Associado a isto, o reconhecimento dos benefícios atrelados ao estoque de carbono das florestas tropicais por meio de políticas internacionais cresce rapidamente, dessa forma, os fundos de incentivo econômico também o fazem (BRANDON, 2014; NORMAN \& NAKHOODA, 2015). Ademais, ressaltase o grande potencial de carbono armazenado na Floresta Amazônica que, de acordo com Soares Filho et al. (2006), equivale a uma década e meia de emissões antropogênicas.

A manutenção das florestas nativas ou ações de reflorestamento visando o sequestro, a conservação, a manutenção e o aumento do estoque e a diminuição do fluxo de carbono são previstos no Código Florestal Brasileiro (Lei 12.651), em que cria a possibilidade da realização de pagamentos à proprietários de terras que assegurem a manutenção de serviços ambientais (BRASIL, 2012). A efetivação de políticas que favoreçam o desenvolvimento social, econômico e ambiental tem grande potencial para estimular a preservação de florestas (BÔAS et al. 2019), bem como a criação de programas de reflorestamento de áreas desmatadas (SANQUETTA et al. 2018), essencialmente em áreas como a região amazônica, onde dados do Instituto Nacional de Pesquisas Espaciais (INPE) mostram que no ano de 2018 a área desmatada foi de $7.536 \mathrm{~km}^{2}$ (INPE, 2019).

Logo, a implantação de projetos envolvendo os PSAs na região contribuiria para frear o desmatamento, além de auxiliar no incremento de renda nas áreas rurais, aumentando o bem-estar das comunidades tradicionais (FASIABEN et al., 2009). Somado ao exposto, Pan et al. (2011) encaram as florestas tropicais em estágio regenerativo, em escala global, como grandes sumidouros de carbono, 1,6 Mg \pm 0,5 Pg C.ano ${ }^{-1}$, de $2000-2007$, com estoques superiores a florestas intactas.

Neste sentido, o desenvolvimento de estudos que possibilitem identificar a quantidade de estoque $e$ incremento de carbono em florestas passa a ser uma ferramenta importante, alicerçando conhecimentos já adquiridos e favorecendo a efetivação do PSA em florestas tropicais.

Portanto, o objetivo deste trabalho foi quantificar a capacidade de sequestro e estocagem de carbono em um remanescente florestal no município de Parauapebas, PA, para fins de Pagamento por Serviço Ambiental.

\section{MATERIAL E MÉTODOS}

O estudo foi desenvolvido em uma parcela permanente de $20 \mathrm{~m}$ x $500 \mathrm{~m}$ (1 ha), inserido em um remanescente de Floresta Ombrófila Densa no campus da Universidade Federal Rural da Amazônia, localizado na cidade de Parauapebas, região sudeste do Pará. O remanescente é caracterizado por ter sofrido alterações devido a ação 
antrópica, como corte exploratório, queimadas eventuais e presença de gado, caracterizando uma floresta secundária.

A área de estudo apresenta, de acordo com a classificação de Köppen, clima do tipo "Awi", tropical chuvoso com seca de inverno, caracterizado por um forte período de estiagem, além de altos valores de precipitação anual (média de $2.236 \mathrm{~mm}$.ano ${ }^{-1}$ ) e temperatura média de 25,4 ㅇ.

Para determinação da quantidade de serapilheira produzida pela floresta foram confeccionados e instalados cinco coletores permanentes, suspensos a um metro do nível do solo, de formato circular, com área conhecida de $1 \mathrm{~m}^{2}$, equidistantes $50 \mathrm{~m}$, no sentido da declividade do terreno (Norte - Sul). O material depositado nos coletores foi coletado quinzenalmente, por um período de um ano (novembro de 2016 a outubro de 2017), sendo posteriormente, separado em componentes: folhas, galhos e miscelânea (flores, sementes, material em avançado estágio de decomposição, entre outros).

As amostras quinzenais foram agrupadas, formando uma única amostra para cada mês. O material coletado foi levado à estufa, onde permaneceu em temperatura de $60 \stackrel{\circ}{\circ} \pm 5$ ㄷ C até alcançar massa constante em três aferições consecutivas. Posteriormente, foram preparadas amostras compostas para cada mês avaliado, seguido pela moagem com auxílio de um moinho do tipo Willey. O teor de carbono foi determinado por meio de um analisador elementar modelo Vario Micro Cube CHNS.

Para avaliar a influência do clima na produção de serapilheira, foram analisados dados referentes a temperatura, umidade e precipitação do Instituto Nacional de Meteorologia (Estação Serra dos Carajás A230) (INMET, 2017). A influência de cada variável na produção de serapilheira foi analisada por meio da correlação de postos de Spearman.

A quantificação do carbono estocado na floresta em pé foi calculada a partir da mensuração dos diâmetros na altura do peito (DAPs), tomados a 1,30 $\mathrm{m}$ do solo, e altura comercial, considerada a altura do solo até a interseção do galho mais grosso no fuste da árvore, de todas as árvores inseridas dentro da parcela do inventário, com diâmetro mínimo de inclusão de $10 \mathrm{~cm}$. A correção do volume cilíndrico dos indivíduos foi feita por meio do fator de forma estabelecido na instrução normativa № 05 da Secretaria de meio Ambiente e Sustentabilidade do estado do Pará, igual a 0,7 (PARÁ, 2011).

A partir do volume calculado, foi utilizado o valor médio de densidade básica da madeira de árvores da região sul da Amazônia $\left(0,59 \mathrm{~g} . \mathrm{cm}^{-3}\right)$ citado por Nogueira et al. (2008), para estimar a massa seca dos indivíduos analisados. A estimava de carbono foi realizada por método indireto, justificado devido ao tempo, custos e extensão das amostras (MARTINS et al., 2017). O valor de massa seca foi multiplicado pelo teor 0,5 para encontrar a massa de carbono presente na biomassa arbórea, justificando um teor médio de carbono de florestas tropicais de 50\%, conforme valor default do Intergovernamental Panel on Climate Change (IPCC, 2001).

Após a quantificação da massa de carbono presente na serapilheira e na floresta em pé, foi realizada a mensuração do valor do estoque de carbono. O valor utilizado como referência correspondeu a commoditie crédito de carbono na bolsa de valores do Reino Unido, cotado a 28,50 €. $\mathrm{Mg}^{-1}$ (LSE, 2019).

\section{RESULTADOS E DISCUSSÃO}

O resultado da análise elementar das amostras compostas da serapilheira mostrou que o carbono representa, em média, $47,85 \%$ da massa seca das folhas, $46,87 \%$ dos galhos e $48,03 \%$ para a miscelânea, resultando em um teor médio geral de carbono para a serapilheira igual a 47,58\%. Segundo Luizão \& Schubart (1987), o carbono representa cerca de $47 \%$ da serapilheira, não havendo grandes diferenças de concentração de carbono entre as diferentes frações do vegetal.

A partir dos teores de carbono de cada componente e na produção de serapilheira durante o período do experimento, determinou-se a quantidade de carbono incorporada à serapilheira, conforme apresentado na Tabela 1.

Tabela 1. Massa de serapilheira e carbono acumulados no remanescente florestal, por componente, durante o período do experimento.

\begin{tabular}{|c|c|c|}
\hline \multirow{2}{*}{ Componente } & Serapilheira & Carbono \\
\hline & \multicolumn{2}{|c|}{ Mg.ha' ${ }^{-1}$} \\
\hline Folha & 3,184 & 1,524 \\
\hline Galho & 1,604 & 0,752 \\
\hline Miscelânea & 0,945 & 0,454 \\
\hline Total & 5,733 & 2,730 \\
\hline
\end{tabular}

Observa-se que a produção total de serapilheira durante o período do experimento foi de 5,733 Mg.ha-1, sendo as folhas o componente que mais contribuiu em massa para este resultado, com 55,54\% do total. Considerando o teor de carbono médio dos componentes da serapilheira, o carbono estocado na serapilheira representou $47,61 \%$ da massa total, sendo 


\section{2,730 Mg.ha-1. ano $^{-1}$.}

$\mathrm{O}$ acúmulo de serapilheira depende de fatores como a cobertura florestal, o estágio sucessional, idade, tipo de floresta, além de fatores como condições edafoclimáticas, regime hídrico, condições climáticas, taxa de decomposição e distúrbios naturais e artificiais (CALDEIRA et al., 2007). Apesar de existirem poucos dados sobre acúmulo de serapilheira em florestas tropicais naturais, a produção de serapilheira observada neste trabalho está de acordo com O'Connell \& Sankaran (1997), que afirmam que em determinados locais da América do Sul, a biomassa de serapilheira acumulada em florestas tropicais naturais varia entre 3,1 e 16,5 Mg.ha-1. ano-1.

A Figura 1 apresenta os valores para temperatura máxima e mínima, precipitação acumulada para o período do experimento.



Precipitação $\longrightarrow$ Temperatura $\left({ }^{\circ} \mathrm{C}\right)$ Max $\longrightarrow$ Temperatura $\left({ }^{\circ} \mathrm{C}\right)$ Min

Figura 1. Variáveis climáticas observadas durante o período do avaliado.

A Tabela 2 apresenta os valores da correlação em percentagem da produção de serapilheira total e por componente com as variáveis climáticas analisadas.

Tabela 2. Valores da correlação da produção de serapilheira total e por componente com as variáveis climáticas analisadas.

\begin{tabular}{cccccc}
\hline \multirow{2}{*}{ Componente } & \multicolumn{2}{c}{ Temperatura } & \multicolumn{2}{c}{ UR\% } & \multirow{2}{*}{ Precipitação } \\
\cline { 2 - 5 } & Máx. & Min. & Máx. & Mín. & \\
\hline Folha & $68 \%$ & $68 \%$ & $-77 \%$ & $-76 \%$ & $-61 \%$ \\
Galho & $30 \%$ & $30 \%$ & $-32 \%$ & $-33 \%$ & $-38 \%$ \\
\hline Miscelânea & $5 \%{ }^{\mathrm{ns}}$ & $4 \%^{\mathrm{ns}}$ & $-3 \%^{\mathrm{ns}}$ & $-3 \%^{\mathrm{ns}}$ & $-7 \%^{\mathrm{ns}}$ \\
\hline Total & $63 \%$ & $63 \%$ & $-67 \%$ & $-67 \%$ & $-56 \%$ \\
\hline
\end{tabular}

Temperatura em graus célsius; UR\% = umidade relativa; Máx. = máximo; Mín. = mínimo; Precipitação = precipitação acumulada $\left(\mathrm{mm} . \mathrm{mês}^{-1}\right){ }^{\text {ns }}=$ correlação não significativa a probabilidade de $95 \%$.

Observa-se que das três frações estudadas, apenas as folhas apresentaram uma forte correlação com as variáveis climáticas (acima de 60\%) e, por ser o principal constituinte da serapilheira, consequentemente a produção total apresentou forte correlação com as variáveis climáticas.

A correlação negativa da massa de folhas e, consequentemente, da serapilheira com a umidade e a precipitação e positiva com a temperatura demonstra que, apesar de a floresta ser composta por espécies perenifólias, estas tendem a reduzir o número de folhas na época da seca, uma característica adaptativa para reduzir a perda de água, já que a maior parte da transpiração ocorre via foliar, evitando assim o estresse hídrico. Sanches et al. (2009) encontraram resultados semelhantes ao avaliarem a dinâmica sazonal da produção e decomposição de serapilheira em floresta tropical de transição. Os autores não encontraram correlação significativa para a temperatura, mas observaram uma correlação negativa significativa entre a produção de serapilheira e umidade relativa do ar e precipitação, indicando uma maior deposição mensal de serapilheira, principalmente de folhas, na estação seca.

$\mathrm{O}$ incremento volumétrico médio anual da floresta analisada foi de 6,71 $\mathrm{m}^{3}$.ha $\mathrm{h}^{-1}$.ano ${ }^{-1}$. Os valores para diâmetro médio, área basal e volume, assim como seus incrementos periódicos anuais (IPA) obtidos a partir do inventário realizado na área são apresentados na Tabela 3.

Tabela 3. Diâmetro médio, área basal, volume e incremento periódico anual encontrados na área estudada.

\begin{tabular}{cccc}
\hline \multirow{2}{*}{ Variáveis } & \multicolumn{2}{c}{ Ano } & \multirow{2}{*}{ IPA } \\
\cline { 2 - 3 } & $\mathbf{2 0 1 6}$ & $\mathbf{2 0 1 8}$ & \\
\hline Diâmetro médio $(\mathrm{cm})$ & 18,74 & 19,53 & 0,39 \\
Área basal $\left(\mathrm{m}^{2} \cdot \mathrm{ha}^{-1}\right)$ & 12,51 & 14,88 & 1,18 \\
Volume $\left(\mathrm{m}^{3} \cdot \mathrm{ha}^{-1}\right)$ & 72,85 & 86,26 & 6,71 \\
\hline
\end{tabular}

O IPA para o diâmetro médio observado neste trabalho é semelhante ao encontrado por Vieira et al. (2005), igual a 0,39 cm e 0,31 cm nas regiões de Rio Branco e Santarém, respectivamente. No entanto, resultados inferiores foram observados por Higushi et al. (2004) e Souza et al. (2012). Higushi et al. (2004), analisando a dinâmica de uma floresta primária da Amazônia Central, obteve IPA em diâmetro de $0,164 \mathrm{~cm}$; Souza et al. (2012), trabalhando com floresta primária na região de Manaus, $A M$, encontrou um IPA em diâmetro de 0,21 cm e 0,13 cm para o período de 2005 a 2007 e 2007 a 2010, respectivamente.

Quanto a área basal, o observado neste trabalho foi superior aos observados na literatura. Oliveira et al. (2005), trabalhando na Floresta Nacional do Tapajós, encontrou incremento de $0,06 \mathrm{~m}^{2}$.ha-1.ano ${ }^{-1}$ para uma área sem 
intervenção. Teixeira et al. (2007) em uma floresta natural de Terra Firme, na região de Manaus, encontrou IPA em área basal de $0,44 \mathrm{~m}^{2}$.ha-1. Souza et al. (2012) trabalhando com floresta primária na região de Manaus, $\mathrm{AM}$, encontrou um incremento em área basal entre $0,33 \mathrm{~m}^{2}$.ha-1 e 0,12 $\mathrm{m}^{2} \cdot \mathrm{ha}^{-1}$.

O IPA em volume observado neste trabalho está próximo ao relatado por Teixeira et al. (2007), que descreveram um incremento em volume de 5,60 $\mathrm{m}^{3}$.ha${ }^{1}$.ano ${ }^{-1}$ para uma floresta de natural de Terra Firme, na região de Manaus. No entanto, valores abaixo ao observado neste estudo foram encontrados por Oliveira et al. (2005), Colpini et al. (2010) e Souza et al. (2012), que ao pesquisarem em florestas naturais ou pouco manejadas relataram IPA em volume igual a $0,70 \mathrm{~m}^{3} \cdot \mathrm{ha}^{-1}, 2,11 \mathrm{~m}^{3}$.ha-1 e 1,31 $\mathrm{m}^{3} \cdot \mathrm{ha}^{-1}$, respectivamente.

Os valores elevados do IPA para as variáveis estudadas, quando comparados com o da literatura para florestas primárias, pode ser justificado devido ao grande número de indivíduos recrutados (que atingem o diâmetro mínimo de inclusão para o inventário) e da baixa mortalidade, resultando em uma taxa de 5,10\% para o recrutamento e 1,40\% para a mortalidade. Para as florestas da Amazônia são relatados na literatura taxas de mortalidade variando entre $0,7 \%$ e $1,3 \%$ e valores entre $0,3 \%$ e $2,0 \%$ para o recrutamento (SOUZA et al., 2012; COLPINI et al., 2010; HIGUSHI et al. 2004). A alta taxa de recrutamento observado no presente estudo é uma situação comum em florestas que sofreram perturbações mais elevadas, pois o aumento no número e/ou tamanho das clareiras e formações florestais secundárias em que há o desenvolvimento das espécies pioneiras, resultam na inclusão de novos indivíduos.

Usando o valor de densidade básica da madeira encontrado por Nogueira et al. (2008), verificou-se que a massa do volume comercial do remanescente é de 50,90

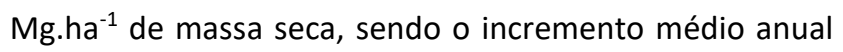
em massa igual a 3,96 Mg.ha-1 ${ }^{-1}$ Fazendo a conversão para massa de carbono, tem-se que a floresta em pé apresenta atualmente 25,45 Mg.ha-1 de carbono estocados e um incremento médio anual de 1,98 Mg.ha-1. O IPA de carbono observado é superior ao encontrado por Higushi et al. 2004, que registrou um incremento médio de carbono de 1,2 Mg.ha- ${ }^{-1}$.ano ${ }^{-1}$ em uma floresta primária na região de Manaus. Atribui-se a diferença entre os valores devido a floresta deste estudo ser secundária, com características diferenciadas quando comparada a do autor supracitado.

Considerando os valores atualmente pagos por tonelada de carbono sequestrado, estima-se que 25,45 Mg.ha-1 de carbono estocado na biomassa viva (volume comercial) representam um total de 725,32 €.ha-1. O carbono sequestrado anualmente totalizou 4,71 Mg.ha-1 (carbono incorporado na serapilheira + incremento médio anual de carbono no volume comercial), totalizando um valor de 134,24 €. ha-1. Somando os dois valores tem-se que o remanescente florestal poderia receber um total de $859,56 €$.ha ${ }^{-1}$ caso entrasse em um programa de pagamento por sequestro e estocagem de carbono.

Semelhante ao que é retratado para a Floresta Amazônica, o grande potencial de recebimento de PSA do remanescente analisado está na manutenção do estoque de carbono da biomassa viva, com $79,18 \%$ do valor total que pode ser recebido, e não no sequestro de carbono propriamente dito.

Isso demonstra que a inserção do remanescente estudado em um programa de PSA apresenta, além das vantagens ambientais, vantagens financeiras em relação a outras formas de uso da terra. Somado a isto, há a possiblidade de mais uma fonte de renda: a exploração dos produtos florestais não madeireiros (PFNM).

Ressalta-se ainda que os valores apresentados dizem respeito apenas ao carbono presente na biomassa viva acima do solo e ao incremento anual serapilheira, não sendo quantificados o carbono presente no solo, na biomassa abaixo do solo, na serapilheira já existente e na copa das árvores, o que gera uma subestimativa do potencial real que a floresta possui para receber créditos de carbono

\section{CONCLUSÕES}

O valor financeiro que pode ser atribuído ao remanescente florestal em um programa de créditos de carbono indica a potencialidade de programas de Pagamento por Serviços Ambientais, que auxiliam na conservação da biodiversidade e apresentar um custo de oportunidade que pode ser superior às demais formas de uso e ocupação da terra.

O carbono armazenado pela floresta secundária é o serviço que contribui majoritariamente para o total de carbono estocado, indicando a importância do correto manejo deste recurso natural, de modo que favoreça o crescimento e processos de ciclagem florestal.

\section{AGRADECIMENTOS}

Ao Conselho Nacional de Desenvolvimento Científico e Tecnológico, CNPq. 


\section{REFERÊNCIAS}

BÔAS, H.V. et al. Influência de gradientes geográficos na estimativa do estoque de carbono em floresta estacional semidecidual. BIOFIX Scientific Journal, v.4, n.2, p.137-145, 2019.

BRANDON, K. Ecosystem services from tropical forests. Review of current science. Center for Global Development Climate and Forest. Washington, 2014.

BRASIL. Lei no 12.651, de 25 de maio de 2012. Diário Oficial da União, Brasília, 2012. Disponível em: http://www.planalto.gov.br/ccivil_03/_Ato2011-2014/2012/Lei /L12651.htm

CALDEIRA, M.V.W. et al. Quantificação de serapilheira e de nutrientes - Floresta Ombrófila Mista Montana - Paraná. Revista Acadêmica, v.5, p.101-116, 2007.

CHAN, K.M.A. et al. Payments for ecosystem services: Rife with problems and potential for transformation towards sustainability. Ecological Economics, v 140, p 110-122, 2017.

COLPINI, C. et al. Incremento, ingresso e mortalidade em uma floresta de contato ombrófila aberta/estacional em Marcelândia, Mato Grosso. Acta Amazonica, v.40, p.549-556, 2010.

FASIABEN, M.C.R. et al. Estimativa de aporte de recursos para um sistema de Pagamento por Serviços Ambientais na floresta Amazônica Brasileira. Ambiente \& Sociedade, v.12, n.2, p.223239, 2009.

HIGUSHI, N. et al. Dinâmica e balanço do carbono da vegetação primária da Amazônia Central. Floresta, v.34, p.295-304, 2004.

INMET - INSTITUTO NACIONAL DE METEOROLOGIA. Banco de dados meteorológicos para ensino e pesquisa. 2019. Disponível em: http://www.inmet.gov.br/portal/index.php?r=bdmep/ bdmep

INPE - INSTITUTO NACIONAL DE PESQUISAS ESPACIAIS. Projeto PRODES. Monitoramento do desmatamento da Floresta Amazônica brasileira por satélite. 2019. Disponível em: http://www.obt.inpe.br/OBT/assuntos/programas/amazonia/pr odes.

IPCC - INTERGOVERNMENTAL PANEL ON CLIMATE CHANGE. Climate change 2001 - Impacts, Adaptation and Vulnerability. Contribution of working group II to the third assessment report of the intergovernmental panel on climate change. New York: Cambridge University Press, 2001.

LSE - LONDON STOCK EXCHANGE. Prices \& markets. 2019. Disponível em: http://www.londonstockexchange.com

LUIZÃO, F.J.; SCHUBART, H.O.R. Produção e decomposição de liteira em floresta de terra firme da Amazônia Central. Acta Limnologica Brasiliensia, v.1, p.575-600, 1987.

MARTINS, A.P.M. et al. Comparação de métodos de quantificação de carbono e, Bracatinga (Mimosa scabrella Benth.). BIOFIX Scientific Journal, v.2, n.1, p.16-21, 2017.
MATTEI, L.; ROSSO, S. Evolução do mercado de pagamento por serviços ecossistêmicos no Brasil: Evidências a partir do setor hídrico. Boletim Regional, Urbano e Ambiental, v.9, p.31-50, 2014.

NOGUEIRA, E.M. et al. Normalization of wood density in biomass estimates of Amazon forests. Forest Ecology and Management, v.256, p.990-996, 2008.

NORMAN, M.; NAKHOODA, S. Current state of REDD+ finance. Center for Global Development Climate and Forest. Washington, 2015.

O'CONNELL, A.M.; SANKARAN, K.V. Organic matter accretion, decomposition and mineralization. Management of soil, nutrients and water in tropical plantations forests. Canberra: ACIAR, 1997.

OLIVEIRA, L.C. et al. Efeito da exploração de madeira e tratamentos silviculturais na composição florística e diversidade de espécies em uma área de 136ha na Floresta Nacional do Tapajós, Belterra, Pará. Scientia Forestalis, v.69, p.62-76, 2005.

OTUKEI, J.R.; EMANUEL, M. Estimation and mapping of above ground biomass and carbon od Bwind impenetrable national park using ALOS PALSAR data. South African Journal of Geomatics, v.4, p.1-13, 2015.

PAN, Y. et al. A large and persistente carbon sink in the world's forest. Science, v.333, p.988-993, 2011.

PARÁ. Instrução Normativa no 05 de 19 de maio de 2011. Dispõe sobre a Política Estadual de Floresta e demais formações de vegetação no Estado do Pará. Pará, 2011. Disponível em: https://www.semas.pa.gov.br/2011/05/19/instrucao-normativano-05-de-19052011/

REIS, M.G.F. et al. Sequestro e armazenamento de carbono em florestas nativas e plantadas dos estados de Minas Gerais e Espírito Santo. Seminário emissão x "sequestro" de $\mathrm{CO}_{2}$ : uma oportunidade de negócios para o Brasil, p.155-195, 1994.

SANCHES, L. et al. Dinâmica sazonal da produção e decomposição de serapilheira em floresta tropical de transição. Revista Brasileira de Engenharia Agrícola e Ambiental, v.13, p.183-189, 2009.

SANQUETTA, C.R. et al. Dinâmica em superfície, volume, biomassa e carbono nas florestas plantadas brasileiras: 19902016. BIOFIX Scientific Journal, v.3, n.1, p.152-160, 2018.

SOARES FILHO, B.S. et al. Modelling conservation the Amazon basin. Nature, v.440, p.520-553, 2006.

SOUZA, C.R. et al. Dinâmica e estoque de carbono em floresta primária na região de Manaus, AM. Acta Amazônica, v.42, p.501506, 2012.

SOUZA, C.R. et al. Projection of diametric distribution and carbono stock of a managed forest in Manaus/AM. Floresta, v.44, p.525-534, 2014.

TEIXEIRA, L. M. et al. Projeção da dinâmica da floresta natural de Terra-firme, região de Manaus-AM, com o uso da cadeia de 
transição probabilística de Markov. Acta Amazonica, v.37, p.377384, 2007.

UN - UNITED NATIONS. MILLENNIUM ECOSYSTEM ASSESSMENT. Ecosystems and Human Being - Synthesis. Washington: Island Press; 2005.

VIEIRA, S.A. et al. Slow growth rates of Amazonian trees: consequences for carbon cycling. PNAS. Proceedings of the National Academy of Sciences of the United States of America, v.102, p.18502-18507, 2005.

WUNDER, S. et al. Pagamentos por serviços ambientais: perspectivas para a Amazônia Legal. 2.ed. Brasília: MMA, 2009.

Recebido em 11-08-2019 Aceito em 16-10-2019 